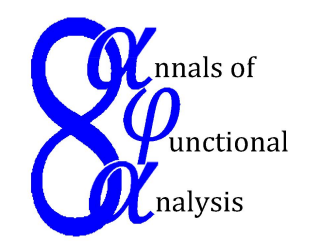

Ann. Funct. Anal. 6 (2015), no. 2, 78-90

http://doi.org/10.15352/afa/06-2-8

ISSN: 2008-8752 (electronic)

http://projecteuclid.org/afa

\title{
ON INEQUALITIES INVOLVING EIGENVALUES AND TRACES OF HERMITIAN MATRICES
}

\author{
RAJESH SHARMA ${ }^{1}$, RAVINDER KUMAR ${ }^{1}$ AND SHALINI GARGA ${ }^{2 *}$ \\ Communicated by Y. Lim
}

\begin{abstract}
It is shown that some immediate consequences of the spectral theorem provide refinements and extensions of the several well-known inequalities involving eigenvalues and traces of Hermitian matrices. We obtain bounds for the spread and condition number of a Hermitian matrix.
\end{abstract}

\section{INTRODUCTION}

Let $\mathbb{M}(n)$ denotes the algebra of all $n \times n$ complex matrices. Let $A=\left(a_{i j}\right)$ be any Hermitian element of $\mathbb{M}(n)$ with eigenvalues $\lambda_{i}$ such that $\lambda_{\min }=\lambda_{1} \leq$ $\lambda_{2} \leq \cdots \leq \lambda_{n}=\lambda_{\max }$. The bounds on eigenvalues in terms of the functions of entries of the given matrix are of great practical importance and have been studied extensively in literature, see $[16,18]$. The inequalities involving eigenvalues of a matrix $A$ and traces of $A^{-1}, A$ and $A^{2}$ are of special interest. It is well-known that

$$
\lambda_{\min } \leq \frac{\operatorname{tr} A}{n} \leq \lambda_{\max }
$$

where $\operatorname{tr} A$ denotes the trace of $A$. The inequality (1.1) is in fact the inequality for numbers, $\operatorname{tr} A=\sum_{i=1}^{n} \lambda_{i}$. It is useful as trace of $A$ can be calculated easily, $\operatorname{tr} A=\sum_{i=1}^{n} a_{i i}$.

Date: Received: May 2, 2014; Accepted: Jul. 1, 2014.

* Corresponding author.

2010 Mathematics Subject Classification. Primary 15A42; Secondary 47A63.

Key words and phrases. Trace, eigenvalue, spread, condition number, Kantorovich inequality. 
If $A$ is a positive definite matrix, then

$$
n^{2} \leq \operatorname{tr} A \operatorname{tr} A^{-1} \leq n^{2} \frac{\left(\lambda_{\min }+\lambda_{\max }\right)^{2}}{4 \lambda_{\min } \lambda_{\max }} .
$$

The inequality (1.2) is the Kantorovich inequality [9] for positive numbers, $\operatorname{tr} A^{-1}=$ $\sum_{i=1}^{n} \lambda_{i}^{-1}$. For a brief history and motivation of the inequality (1.2), see [14]. Similarly, the Krasnoselskii and Krein inequality [10] for positive numbers gives

$$
\frac{1}{n} \leq \frac{\operatorname{tr} A^{2}}{(\operatorname{tr} A)^{2}} \leq \frac{1}{n} \frac{\left(\lambda_{\min }+\lambda_{\max }\right)^{2}}{4 \lambda_{\min } \lambda_{\max }} .
$$

Further, the spread of $A$ is defined as

$$
\operatorname{spd}(A)=\max _{i, j}\left|\lambda_{i}-\lambda_{j}\right| \text {. }
$$

The idea of spread was proposed by Mirsky [12] and says that the distance between any two eigenvalues is at most equal to the spread. Beginning with Mirsky [12] several authors have obtained bounds for the spread of a matrix; see [1, 4-8, $11-13,17-18]$. A lower bound for the spread in terms of traces of $A$ and $A^{2}$ is

$$
\operatorname{spd}(A) \geq 2 \sqrt{\frac{\operatorname{tr} A^{2}}{n}-\left(\frac{\operatorname{tr} A}{n}\right)^{2}} .
$$

See $[3,18]$. The inequality (1.4) is an immediate consequence of an upper bound for the variance of $n$ real numbers due to Popoviciu [15].

For a positive definite matrix $A$, the ratio of the largest to the smallest eigenvalue is called the condition number of $A$. Wolkowicz and Styan [18] have shown that

$$
\frac{\lambda_{\max }}{\lambda_{\min }} \geq 1+\frac{2 s}{\frac{\operatorname{tr} A}{n}-\frac{s}{\sqrt{n-1}}},
$$

where $s^{2}=\frac{1}{n} \operatorname{tr} A^{2}-\left(\frac{\operatorname{tr} A}{n}\right)^{2}$.

All the above inequalities are essentially the inequalities for real numbers. We here show that some immediate consequences of the spectral theorem give some further extensions of the above inequalities for matrices. The spectral theorem says that for every normal matrix $A \in \mathbb{M}(n)$ there exists a unitary matrix $U \in$ $\mathbb{M}(n)$ such that $U^{*} A U=\operatorname{diag}\left(\lambda_{1}, \lambda_{2}, \cdots, \lambda_{n}\right)=D$. So,

$$
A=U D U^{*}=\sum_{i=1}^{n} \lambda_{i} P_{i},
$$

where $P_{i}=u_{i} u_{i}^{*}, u_{i}$ is the $i^{\text {th }}$ column of $U$. Then, $P_{i}^{2}=P_{i}=P_{i}^{*}, P_{i} P_{j}=0$ for $i \neq j$ and $\sum_{i=1}^{n} P_{i}=I$. Let $f$ be a real function define on an interval containing the eigenvalues of $A$. We define $f(D)=\operatorname{diag}\left(f\left(\lambda_{1}\right), f\left(\lambda_{2}\right), \cdots, f\left(\lambda_{n}\right)\right)$, and $f(A)=U f(D) U^{*}$. See [2]. 
We first prove some basic results for the projections and Hermitian matrices, and use these results in the proofs of the subsequent theorems, (Lemma 2.1-2.5, below). An improvement of the inequality (1.1) is obtained, (Theorem 2.6). Refinements and extensions of the inequalities (1.2) and (1.3) are given, (Theorem 2.7-2.8). Lower bounds for the spread are derived for Hermitian matrices, (Theorem 2.9). We prove a lower bound for the condition number, (Theorem 2.10). It is shown that similar arguments give bounds for the extreme eigenvalues and provide a refinement of the Hadamard inequality for positive definite matrices, (Theorem 2.11-2.12, Remark 2.13). A bound for the eigenvalue of arbitrary matrix is obtained, (Theorem 2.15). We compare our bounds with those given in literature, (Example 1-2).

\section{MAin RESUlts}

Lemma 2.1. Let $A \in \mathbb{M}(n)$ be a Hermitian matrix. If $A^{2} \leq A$, then

$$
\sum_{\substack{j=1 \\ i \neq j}}^{n}\left|a_{i j}\right|^{2} \leq \frac{1}{4}
$$

for all $i=1,2, \cdots, n$. If $A^{2}=A$, then

$$
\sum_{i, j}^{n} a_{i j} \geq 0
$$

Proof. The diagonal entries of $A^{2}$ are $\sum_{j=1}^{n}\left|a_{i j}\right|^{2}, i=1,2, \cdots, n$. Since $A^{2} \leq A$, $\sum_{j=1}^{n}\left|a_{i j}\right|^{2} \leq a_{i i}$. Therefore,

$$
\sum_{\substack{j=1 \\ i \neq j}}^{n}\left|a_{i j}\right|^{2} \leq a_{i i}\left(1-a_{i i}\right)
$$

The inequality (2.1) follows from (2.2) and the fact that the inequality $x(1-x) \leq$ $\frac{1}{4}$ holds for every real number $x$. Further, if $A^{2}=A$,

$$
0 \leq \sum_{i=1}^{n}\left|\sum_{k=1}^{n} a_{i k}\right|^{2}=\sum_{i=1}^{n} b_{i i}+2 \operatorname{Re} \sum_{i>j}^{n} b_{i j}=\sum_{i, j}^{n} b_{i j}=\sum_{i, j}^{n} a_{i j}
$$

where $b_{i j}$ is the $(i, j)^{\text {th }}$ entry of $A^{2}$.

Lemma 2.2. Let $A_{i} \in \mathbb{M}(n)$ be positive semidefinite matrices, $i=1,2, \cdots, n$. Let $a_{i, r s}$ be the $(r, s)^{\text {th }}$ entry of $A_{i}$. If $\sum_{i=1}^{n} a_{i, r r}=1, r=1,2, \cdots, n$, then

$$
\sum_{i=1}^{n}\left|a_{i, r s}\right| \leq 1
$$


Proof. For $A_{i} \geq 0$, we have

$$
\left|a_{i, r s}\right| \leq \sqrt{a_{i, r r} a_{i, s s}} \leq \frac{a_{i, r r}+a_{i, s s}}{2} .
$$

So,

$$
\sum_{i=1}^{n}\left|a_{i, r s}\right| \leq \frac{1}{2}\left(\sum_{i=1}^{n} a_{i, r r}+\sum_{i=1}^{n} a_{i, s s}\right)=1 .
$$

Lemma 2.3. Let $P_{i} \in \mathbb{M}(n)$ be orthogonal projections such that $\sum_{i=1}^{n} P_{i}=I$. Let $p_{i, r s}$ be the $(r, s)^{\text {th }}$ entry of $P_{i}$. Then,

$$
\begin{aligned}
\text { (1) } \sum_{i=1}^{n} p_{i, r r} & =1, \quad \text { (2) } \sum_{\substack{s=1 \\
r \neq s}}^{n} p_{i, r s}=0, \quad \text { (3) } \sum_{\substack{s=1 \\
r \neq s}}^{n}\left|p_{i, r s}\right|^{2} \leq \frac{1}{4} \\
\text { (4) } \quad\left|p_{i, r s}\right| & \leq \frac{1}{2} \text { for } r \neq s \quad \text { and } \quad \text { (5) } \sum_{\substack{s=1 \\
r \neq s}}^{n}\left|p_{i, r s}\right| \leq 1 .
\end{aligned}
$$

Proof. The diagonal and non-diagonal entries of $\sum_{i=1}^{n} P_{i}$ are respectively $\sum_{i=1}^{n} p_{i, r r}$ and $\sum_{i=1}^{n} p_{i, r s}$. So, (1) and (2) follow immediately from $\sum_{i=1}^{n} P_{i}=I$. The inequalities (3) and (5) follow respectively from Lemma 2.1 and Lemma 2.2. The inequality (3) implies (4).

Lemma 2.4. Let $A$ be a normal matrix. Let $b_{r s}$ be the $(r, s)^{\text {th }}$ entry of $f(A)$, then

$$
b_{r r}=\sum_{i=1}^{n} f\left(\lambda_{i}\right) p_{i, r r} \quad \text { and } \quad b_{r s}=\sum_{i=1}^{n} f\left(\lambda_{i}\right) p_{i, r s}
$$

where $p_{i, r r}$ are non-negative real numbers such that $\sum_{i=1}^{n} p_{i, r r}=1$ and $p_{i, r s}$ are complex numbers such that $\sum_{i=1}^{n} p_{i, r s}=0$.

Proof. The eigenvalues of $f(A)$ are $f\left(\lambda_{i}\right), i=1,2, \cdots, n$. It follows from (1.6) that

$$
f(A)=\sum_{i=1}^{n} f\left(\lambda_{i}\right) P_{i} .
$$

Let $p_{i, r s}$ be the $(r, s)^{t h}$ entry of $P_{i}$. The assertions of the Lemma now follow easily on using Lemma 2.3 .

Lemma 2.5. Let $A \in \mathbb{M}(n)$ be a Hermitian matrix. Then, there is a unitary matrix $U \in \mathbb{M}(n)$ such that one of the non-diagonal entry of $U^{*} A U$ is $\left|a_{r s}\right|$, where $a_{r s}$ is the $(r, s)^{\text {th }}$ entry of $A$. 
Proof. Suppose that $U_{r r}=e^{\frac{i \theta}{2}}, U_{s s}=e^{\frac{-i \theta}{2}}$ and $U_{j j}=1$ for $j \neq r, s$. Choose $U=\operatorname{diag}\left(U_{11}, U_{22}, \cdots, U_{n n}\right)$. A simple calculation shows that the $(r, s)^{t h}$ entry of $U^{*} A U$ is $\left|a_{r s}\right|$.

Theorem 2.6. For any Hermitian element $A$ of $\mathbb{M}(n)$, the inequalities

$$
\lambda_{\min }+\frac{2}{n}\left|a_{r s}\right| \leq \frac{\operatorname{tr} A}{n} \leq \lambda_{\max }-\frac{2}{n}\left|a_{r s}\right|,
$$

hold for $r \neq s$.

Proof. By Lemma 2.5, it is sufficient to prove the theorem for the case when $(r, s)^{t h}$ entry of $A$ is $\left|a_{r s}\right|$. By Lemma 2.4,

$$
\left|a_{r s}\right|=\sum_{i=1}^{n} \lambda_{i} p_{i, r s} \text { and }\left|a_{s r}\right|=\sum_{i=1}^{n} \lambda_{i} \overline{p_{i, r s}}
$$

where $p_{i, r s}$ are complex numbers such that $\sum_{i=1}^{n} p_{i, r s}=0, r \neq s$. It is clear from Lemma 2.3 that $\left|p_{i, r s}\right| \leq \frac{1}{2}$. Since $\left|a_{r s}\right|=\left|a_{s r}\right|$, therefore $\left|a_{r s}\right|=\sum_{i=1}^{n} \lambda_{i} g_{i}$ where $g_{i}=\frac{p_{i, r s}+\overline{p_{i, r s}}}{2}$ are real numbers such that $\sum_{i=1}^{n} g_{i}=0$ and $\left|g_{i}\right|=\left|\operatorname{Re} p_{i, r s}\right| \leq \frac{1}{2}$. Let

$$
c_{i}=\frac{1 \pm 2 g_{i}}{n} .
$$

It is easily seen that $0 \leq c_{i} \leq \frac{2}{n} \leq 1$ and $\sum_{i=1}^{n} c_{i}=1$. Also,

$$
\sum_{i=1}^{n} c_{i} \lambda_{i}=\frac{1}{n} \sum_{i=1}^{n} \lambda_{i} \pm \frac{2}{n} \sum_{i=1}^{n} \lambda_{i} g_{i}=\frac{\operatorname{tr} A}{n} \pm \frac{2}{n}\left|a_{r s}\right| .
$$

The inequality (2.3) follows from (2.5) and the fact that $\lambda_{\min } \leq \sum_{i=1}^{n} c_{i} \lambda_{i} \leq$ $\lambda_{\max }$.

Note that the inequalities (2.3) give bounds for the extreme eigenvalues,

$$
\lambda_{\max } \geq \frac{\operatorname{tr} A}{n}+\frac{2}{n}\left|a_{r s}\right| \quad \text { and } \quad \lambda_{\min } \leq \frac{\operatorname{tr} A}{n}-\frac{2}{n}\left|a_{r s}\right| .
$$

We now prove an extension of the Kantorovich inequality (1.2). We denote the $(r, s)^{t h}$ entry of $A, A^{2}$ and $A^{-1}$ respectively by $a_{r s}, b_{r s}$ and $c_{r s}$. Also, $\alpha=\operatorname{Re} a_{r s}$ (or $\left.\operatorname{Im} a_{r s}\right), \beta=\operatorname{Re} b_{r s}\left(\right.$ or $\left.\operatorname{Im} b_{r s}\right)$ and $\gamma=\operatorname{Re} c_{r s}$ (or $\operatorname{Im} c_{r s}$ ). We prove the following theorems for the case when $\alpha, \beta$ and $\gamma$ are respectively the real parts of the corresponding entries. The arguments are similar for the case when we consider the imaginary parts of the entries.

Theorem 2.7. For every positive definite matrix $A \in \mathbb{M}(n)$, we have

$$
n^{2} \leq(\operatorname{tr} A \pm 2 \alpha)\left(\operatorname{tr} A^{-1} \pm 2 \gamma\right) \leq n^{2} \frac{\left(\lambda_{\min }+\lambda_{\max }\right)^{2}}{4 \lambda_{\min } \lambda_{\max }} .
$$


Proof. For $n$ positive real numbers $x_{i}, i=1,2, \cdots, n$, we have

$$
1 \leq\left(\sum_{i=1}^{n} c_{i} x_{i}\right)\left(\sum_{i=1}^{n} \frac{c_{i}}{x_{i}}\right) \leq \frac{\left(x_{\min }+x_{\max }\right)^{2}}{4 x_{\min } x_{\max }}
$$

where $c_{i}$ are non-negative real numbers such that $\sum_{i=1}^{n} c_{i}=1$, see [9]. As in the proof of Theorem 2.6, we have

$$
\sum_{i=1}^{n} \frac{c_{i}}{\lambda_{i}}=\frac{\operatorname{tr} A^{-1}}{n} \pm \frac{2}{n} \gamma
$$

where $c_{i}$ are given in (2.4). Combine (2.5), (2.7) and (2.8), we immediately get (2.6).

Theorem 2.8. Under the conditions of Theorem 2.7, we have

$$
\frac{1}{n} \leq \frac{\operatorname{tr} A^{2} \pm 2 \beta}{(\operatorname{tr} A \pm 2 \alpha)^{2}} \leq \frac{1}{n} \frac{\left(\lambda_{\min }+\lambda_{\max }\right)^{2}}{4 \lambda_{\min } \lambda_{\max }} .
$$

Proof. For $n$ positive real numbers $x_{i}, i=1,2, \cdots, n$, we have

$$
1 \leq \frac{\sum_{i=1}^{n} c_{i} x_{i}^{2}}{\left(\sum_{i=1}^{n} c_{i} x_{i}\right)^{2}} \leq \frac{\left(x_{\min }+x_{\max }\right)^{2}}{4 x_{\min } x_{\max }},
$$

where $c_{i}$ are non-negative real numbers such that $\sum_{i=1}^{n} c_{i}=1$. We have

$$
\sum_{i=1}^{n} c_{i} \lambda_{i}^{2}=\frac{\operatorname{tr} A^{2}}{n} \pm \frac{2}{n} \beta,
$$

where $c_{i}$ are given in (2.4). Combine (2.5), (2.10) and (2.11), we get (2.9).

Theorem 2.9. For any Hermitian element $A$ of $\mathbb{M}(n)$,

$$
\operatorname{spd}(A) \geq 2\left(\frac{\operatorname{tr} A^{2} \pm 2 \beta}{n}-\left(\frac{\operatorname{tr} A \pm 2 \alpha}{n}\right)^{2}\right)^{\frac{1}{2}} .
$$

Proof. For $n$ real numbers $x_{i}, i=1,2, \cdots, n$, we have [4]

$$
\sum_{i=1}^{n} c_{i} x_{i}^{2}-\left(\sum_{i=1}^{n} c_{i} x_{i}\right)^{2} \leq\left(\frac{x_{\max }-x_{\min }}{2}\right)^{2}
$$

where $c_{i}$ are non-negative real numbers such that $\sum_{i=1}^{n} c_{i}=1$. Combine (2.5), (2.11) and (2.13), we get (2.12).

We now prove a lower bound for the condition number of a positive definite matrix. 
Theorem 2.10. For any positive definite matrix $A \in \mathbb{M}(n)$, we have

$$
\frac{\lambda_{\max }}{\lambda_{\min }} \geq\left(\frac{b}{a}+\sqrt{1+\left(\frac{b}{a}\right)^{2}}\right)^{2}
$$

where

$$
a=\frac{\operatorname{tr} A \pm 2 \alpha}{n} \text { and } b=\sqrt{\frac{\operatorname{tr} A^{2} \pm 2 \beta}{n}-a^{2}} .
$$

Proof. The inequality (2.14) follows from the fact that for $n$ positive real numbers $x_{i}, i=1,2, \cdots, n$, we have [5]

$$
\frac{x_{\max }}{x_{\min }} \geq\left(\frac{\sqrt{\sum_{i=1}^{n} c_{i} x_{i}^{2}-\left(\sum_{i=1}^{n} c_{i} x_{i}\right)^{2}}}{\sum_{i=1}^{n} c_{i} x_{i}}+\sqrt{1+\frac{\sum_{i=1}^{n} c_{i} x_{i}^{2}-\left(\sum_{i=1}^{n} c_{i} x_{i}\right)^{2}}{\left(\sum_{i=1}^{n} c_{i} x_{i}\right)^{2}}}\right)^{2},
$$

where $c_{i}$ are non-negative real numbers such that $\sum_{i=1}^{n} c_{i}=1$.

A simple lower bound for the largest eigenvalue of a positive definite matrix $A$ is

$$
\lambda_{\max } \geq \frac{\operatorname{tr} A^{2}}{\operatorname{tr} A} .
$$

We prove an extension of this inequality in the following theorem.

Theorem 2.11. For every positive definite matrix $A \in \mathbb{M}(n)$, we have

$$
\lambda_{\max } \geq \frac{\operatorname{tr} A^{2} \pm 2 \beta}{\operatorname{tr} A \pm 2 \alpha} .
$$

Proof. It is evident that for $n$ positive real numbers $x_{i}, i=1,2, \cdots, n$, we have

$$
x_{\max } \geq \frac{\sum_{i=1}^{n} c_{i} x_{i}^{2}}{\sum_{i=1}^{n} c_{i} x_{i}},
$$

where $0 \leq c_{i} \leq 1$ and $\sum_{i=1}^{n} c_{i}=1$. Combine (2.5), (2.11) and (2.16), we get $(2.15)$.

Note that

$$
\frac{1}{n} \sum_{i, j}^{n} a_{i j}=\sum_{i}^{n} \lambda_{i} q_{i} \text { where } q_{i}=\frac{1}{n} \sum_{r, s}^{n} p_{i, r s}
$$

and $p_{i, r s}$ is the $(r, s)^{t h}$ entry of projection $P_{i}$. It is clear that $\sum_{i}^{n} q_{i}=1$ and by Lemma $2.1, q_{i} \geq 0$. So, we have the well-known inequality,

$$
\lambda_{\min } \leq \frac{1}{n} \sum_{i, j}^{n} a_{i j}=\frac{\operatorname{tr} A}{n}+\frac{1}{n} \sum_{i \neq j}^{n} a_{i j} \leq \lambda_{\max } .
$$

We show that the above arguments also provide an extension of the inequality (2.17). This also gives an alternative proof of the Theorem 2.6. We use Weyl's 
theorem which says that if $\lambda_{i}(A), \lambda_{i}(B)$ and $\lambda_{i}(A+B)$ are respectively the eigenvalues of $A, B$ and $A+B$ in increasing order, then for all $k=1,2, \cdots, n$, we have

$$
\lambda_{k}(A)+\lambda_{1}(B) \leq \lambda_{k}(A+B) \leq \lambda_{k}(A)+\lambda_{n}(B)
$$

See $[2]$.

Theorem 2.12. Let $A \in \mathbb{M}(n)$ be a real symmetric matrix. Then

$$
\lambda_{\min } \leq \frac{\operatorname{tr} A}{n}+\frac{2}{n} \sum_{\substack{i<j \\ i \notin I}}^{n} a_{i j} \leq \lambda_{\max }
$$

where $I \subset\{1,2, \cdots, n\}$.

Proof. Let $A_{1}=U_{k}^{*} A U_{k}$ where $U_{k}=\operatorname{diag}\left(U_{11}, U_{22}, \cdots, U_{n n}\right), U_{k k}=-1$ and $U_{i i}=$ 1 for $i=1,2, \cdots, n$ and $i \neq k$. Then, the non-diagonal entries of $k^{\text {th }}$ row and column of $A+A_{1}$ are all zero. It follows from Weyl's inequality (2.18) that

$$
\lambda_{\max }(A) \geq \lambda_{\max }\left(\frac{A+A_{1}}{2}\right) \geq \frac{\operatorname{tr} A}{n}+\frac{2}{n} \sum_{\substack{i<j \\ i \neq k}}^{n} a_{i j} .
$$

Now, let $A_{2}=U_{l}^{*} A_{1} U_{l}$ where $l \neq k$. The non-diagonal entries of $k^{\text {th }}$ and $l^{\text {th }}$ row and column of $A_{1}+A_{2}$ are all zero. We therefore have

$$
\lambda_{\max }(A) \geq \lambda_{\max }\left(\frac{A+A_{1}+A_{2}}{2}\right) \geq \frac{\operatorname{tr} A}{n}+\frac{2}{n} \sum_{\substack{i<j \\ i \neq k, l}}^{n} a_{i j} .
$$

The process can be repeated, and we conclude that the second inequality (2.19) holds good. The first inequality (2.19) follows on using similar arguments.

It follows from the proof of the above theorem that $\lambda_{\max }(A) \geq \lambda_{\max }(X)$ where all the non-diagonal entries of $X$ except one $(r, s)^{t h}$ entry $a_{r s}$ is non-zero. It is clear that $X$ is unitarily similar to $Y$ whose $(r, s)^{t h}$ entry is $\left|a_{r s}\right|$. The inequalities (2.3) then follow from (2.19), use Lemma 2.5. It is worthwhile here to note one more alternative proof of inequality (2.3). We have

$$
\begin{aligned}
\left|a_{r s}\right| & =\left|\sum_{i=1}^{n} \lambda_{i} p_{i, r s}\right| \leq\left|\sum_{i=1}^{n}\left(\lambda_{i}-\frac{\lambda_{\max }+\lambda_{\min }}{2}\right) p_{i, r s}\right| \\
& \leq \sum_{i=1}^{n}\left|\lambda_{i}-\frac{\lambda_{\max }+\lambda_{\min }}{2}\right|\left|p_{i, r s}\right| \leq \frac{\lambda_{\max }-\lambda_{\min }}{2}
\end{aligned}
$$

From (2.20), we have

$$
\lambda_{\max } \geq \lambda_{\min }+2\left|a_{r s}\right| .
$$


Add $\lambda_{2}+\lambda_{3}+\cdots+\lambda_{\max }$ on both sides of (2.21) and divide by $n$, we get that

$$
\lambda_{\max } \geq \frac{2 \lambda_{\max }+\lambda_{2}+\lambda_{3}+\cdots+\lambda_{n-1}}{n} \geq \frac{\operatorname{tr} A}{n}+\frac{2}{n}\left|a_{r s}\right| .
$$

Similarly, we have

$$
\lambda_{\min } \leq \frac{2 \lambda_{\min }+\lambda_{2}+\lambda_{3}+\cdots+\lambda_{\max }}{n} \leq \frac{\operatorname{tr} A}{n}-\frac{2}{n}\left|a_{r s}\right| .
$$

Remark 2.13. Let $A$ be as in Theorem 2.6. If diagonal entries of $A$ are all equal, then

$$
\lambda_{\max } \geq a_{11}+\max _{r \neq s}\left|a_{r s}\right|
$$

and

$$
\lambda_{\min } \leq a_{11}-\min _{r \neq s}\left|a_{r s}\right| .
$$

Apply Theorem 2.6 to any $2 \times 2$ principal submatrix of $A$ containing diagonal entries. We immediately get (2.22) and (2.23).

In this connection, it is worthwhile to note the analogous inequalities for the case when diagonal entries are not necessary equal. Under the conditions of Theorem 2.6, we have

$$
\lambda_{\max } \geq \max \left\{\min _{\substack{i=r, s \\ r \neq s}} a_{i i}+\left|a_{r s}\right|\right\}
$$

and

$$
\lambda_{\min } \leq \min \left\{\max _{\substack{i=r, s \\ r \neq s}} a_{i i}-\left|a_{r s}\right|\right\} .
$$

Note that the largest eigenvalue of $A$ is greater than or equal to the largest eigenvalue of any $2 \times 2$ principal submatrix of $A$. The largest eigenvalue of $\begin{aligned} & {\left[\begin{array}{ll}a_{r r} & a_{r s} \\ \overline{a_{r s}} & a_{s s}\end{array}\right] \text { is } } \\ & \mu=\frac{1}{2}\left(a_{r r}+a_{s s}+\sqrt{\left(a_{r r}-a_{s s}\right)^{2}+4\left|a_{r s}\right|^{2}}\right) \geq \min _{\substack{i=r, s \\ r \neq s}} a_{i i}+\left|a_{r s}\right| .\end{aligned}$

It may be noted here that the arguments in the proof of Theorem 2.12 also provide an alternative proof and a refinement of the Hadamard inequality for positive definite matrices. The Minkowski inequality states that if $A_{i} \geq 0, i=$ $1,2, \cdots, n$, then

$$
\left(\operatorname{det}\left(A_{1}+A_{2}\right)\right)^{\frac{1}{n}} \geq\left(\operatorname{det} A_{1}\right)^{\frac{1}{n}}+\left(\operatorname{det} A_{2}\right)^{\frac{1}{n}} .
$$

Therefore, if $A_{1}$ and $A_{2}$ are similar matrices, then

$$
\operatorname{det}\left(\frac{A_{1}+A_{2}}{2}\right) \geq \operatorname{det} A_{1} \text {. }
$$


Let $A=A_{0}$ and

$$
A_{k}=\frac{A_{k-1}+U_{k}^{*} A_{k-1} U_{k}}{2}, k=1,2, \cdots, n
$$

where $U_{k}=\operatorname{diag}\left(U_{11}, U_{22}, \cdots, U_{n n}\right), U_{k k}=-1$ and $U_{j j}=1$ for $j \neq k, k=$ $1,2, \cdots, n$. On using (2.24), we see that $\operatorname{det} A \leq \operatorname{det} A_{1} \leq \cdots \leq \operatorname{det} A_{n-1}$. The inequality $\operatorname{det} A \leq \operatorname{det} A_{n-1}=a_{11} a_{22} \cdots a_{n n}$ gives Hadamard's inequality. It is also clear that $\operatorname{det} A \leq \operatorname{det} B$ where $(r, s)^{\text {th }}$ entry of $B$ is $\left|a_{r s}\right|$ and all other non-diagonal entries are zero. We therefore have

$$
\operatorname{det} A \leq \prod_{i=1}^{n} a_{i i}-\prod_{i \neq r, s}^{n} a_{i i}\left|a_{r s}\right|^{2},
$$

for all $r \neq s$. This provides a refinement of Hadamard's inequality.

We now show that an inequality involving eigenvalues of arbitrary matrix follows from an inequality for complex numbers.

Let $z_{1}, z_{2}, \cdots, z_{n}$ denote $n$ complex numbers. Their arithmetic mean and variance are respectively defined as

$$
\widetilde{z}=\frac{1}{n} \sum_{i=1}^{n} z_{i}
$$

and

$$
s^{2}=\frac{1}{n} \sum_{i=1}^{n}\left|z_{i}-\widetilde{z}\right|^{2}=\frac{1}{n} \sum_{i=1}^{n}\left|z_{i}\right|^{2}-|\widetilde{z}|^{2} .
$$

Lemma 2.14. With notations as above, the inequality

$$
s^{2} \geq \frac{(j-1)(n-j+1)}{n^{2}}\left|z_{j}-z_{j-1}\right|^{2},
$$

holds good for some permutation of numbers $z_{i}, i=1,2,3, \cdots, n$.

Proof. On using the Lagrange identity, we have

$$
s^{2}=\frac{1}{n^{2}} \sum_{i<j}^{n}\left|z_{i}-z_{j}\right|^{2} .
$$

It is clear that for some permutation of numbers $z_{i}$, we have $\left|z_{i}-z_{1}\right| \geq\left|z_{2}-z_{1}\right|$, $i=2, \cdots, n$. Therefore

$$
\sum_{i=2}^{n}\left|z_{i}-z_{1}\right|^{2} \geq(n-1)\left|z_{2}-z_{1}\right|^{2} .
$$

It follows from (2.26) and (2.27) that (2.25) is true for $j=2$. For the general case, there is a permutation of numbers $z_{i}$ such that $\left|z_{i}-z_{k}\right| \geq\left|z_{j}-z_{j-1}\right|, \quad i=$ 
$j, j+1, \cdots, n$ and $k=1,2, \cdots, j-1$, and therefore

$\sum_{i=j}^{n}\left|z_{i}-z_{1}\right|^{2}+\sum_{i=j}^{n}\left|z_{i}-z_{2}\right|^{2}+\cdots+\sum_{i=j}^{n}\left|z_{i}-z_{j-1}\right|^{2} \geq(j-1)(n-j+1)\left|z_{j}-z_{j-1}\right|^{2}$,

for all $j=2,3, \cdots, n$. The inequality (2.25) follows from (2.26) and (2.28).

Theorem 2.15. Let $A \in \mathbb{M}(n)$ be any arbitrary matrix with at least two distinct eigenvalues. Let $\|A\|_{2}$ denotes the Frobenius norm of $A$. If $\lambda_{k}$ is any eigenvalue of $A$, then the disk

$$
\left|\lambda-\lambda_{k}\right| \leq \frac{n}{\sqrt{n-1}} \sqrt{\frac{\|A\|_{2}^{2}}{n}-\left|\frac{\operatorname{tr} A}{n}\right|^{2}}
$$

contains one more eigenvalue of $A$ other than $\lambda_{k}$.

Proof. Let $\widetilde{z_{\lambda}}$ and $s_{\lambda}^{2}$ respectively denotes the arithmetic mean and variance of the eigenvalues $\lambda_{1}, \lambda_{2}, \cdots, \lambda_{n}$,

$$
\widetilde{z_{\lambda}}=\frac{1}{n} \sum_{i=1}^{n} \lambda_{i}=\frac{\operatorname{tr} A}{n}
$$

and

$$
s_{\lambda}^{2}=\frac{1}{n} \sum_{i=1}^{n}\left|\lambda_{i}-\widetilde{z_{\lambda}}\right|^{2}=\frac{1}{n} \sum_{i=1}^{n}\left|\lambda_{i}\right|^{2}-\left|\frac{1}{n} \sum_{i=1}^{n} \lambda_{i}\right|^{2}
$$

Also,

$$
\sum_{i=1}^{n}\left|\lambda_{i}\right|^{2} \leq\|A\|_{2}^{2}
$$

From (2.30) and (2.31), we have

$$
s_{\lambda}^{2} \leq \frac{\|A\|_{2}^{2}}{n}-\left|\frac{\operatorname{tr} A}{n}\right|^{2} .
$$

It follows from Lemma 2.14 that

$$
\left|\lambda_{j}-\lambda_{j-1}\right|^{2} \leq \frac{n^{2}}{(j-1)(n-j+1)} s_{\lambda}^{2},
$$

$j=2,3, \cdots, n$. The right hand side expression in (2.33) is maximum at $j=2$ and $j=n$. We conclude that if $\lambda_{k}$ is any eigenvalue then there is an eigenvalue $\lambda$ such that

$$
\left|\lambda-\lambda_{k}\right|^{2} \leq \frac{n^{2}}{n-1} s_{\lambda}^{2}
$$

Combine (2.32) and (2.34); we immediately get (2.29). 
Example 2.16. Let

$$
A=\left[\begin{array}{lll}
2 & 1 & 2 \\
1 & 1 & 1 \\
2 & 1 & 3
\end{array}\right], \quad A^{-1}=\left[\begin{array}{ccc}
2 & -1 & -1 \\
-1 & 2 & 0 \\
-1 & 0 & 1
\end{array}\right]
$$

Then

$\operatorname{tr} A \operatorname{tr} A^{-1}=30,\left(\operatorname{tr} A+2 a_{23}\right)\left(\operatorname{tr} A^{-1}+2 c_{23}\right)=35,\left(\operatorname{tr} A-2 a_{23}\right)\left(\operatorname{tr} A^{-1}-2 c_{23}\right)=$ 14. So, the inequality (2.6) gives better estimate than the Kantorovich inequality (1.2). Also, $\frac{\operatorname{tr} A^{2}}{(\operatorname{tr} A)^{2}}=0.7222, \frac{\operatorname{tr} A^{2}-2 b_{13}}{\left(\operatorname{tr} A-2 a_{13}\right)^{2}}=1$ and $\frac{\operatorname{tr} A^{2}+2 b_{13}}{\left(\operatorname{tr} A+2 a_{13}\right)^{2}}=0.48$. So, (2.9) gives better estimate than (1.3). From (1.4), $\operatorname{spd}(A) \geq 4.3205$ while from (2.12) $\operatorname{spd}(A) \geq 4.714, a_{23}=1$.

Example 2.17. We now compare our bounds with the corresponding bounds given by Wolkowicz and Styan [18]. It is shown in [18] that for the matrix

$$
A=\left[\begin{array}{llll}
4 & 0 & 2 & 3 \\
0 & 5 & 0 & 1 \\
2 & 0 & 6 & 0 \\
3 & 1 & 0 & 7
\end{array}\right]
$$

$\lambda_{4} \geq 7.158$. From (2.15), $\lambda_{4} \geq 7.8571$. The lower bound for the condition number from (1.5) and (2.14) are 2.4953 and 3.5551, respectively

Acknowledgement. The authors are grateful to Prof. Rajendra Bhatia for the useful discussions and suggestions. The first two authors thank I.S.I. Delhi for a visit in January 2014 when this work had begun.

\section{REFERENCES}

1. E.R. Barnes, A.J. Hoffman, Bounds for the spectrum of normal matrices, Linear Algebra Appl. 201 (1994), 79-90.

2. R. Bhatia, Matrix Analysis, Springer Verlag New York, 2000.

3. R. Bhatia, Positive Definite Matrices, Princeton University Press, 2007.

4. R. Bhatia and C. Davis, A better bound on the variance, Amer. Math. Monthly 107 (2000), 353-357.

5. R. Bhatia and R. Sharma, Some inequalities for positive linear maps, Linear Algebra Appl. 436 (2012), 1562-1571.

6. R. Bhatia and R. Sharma, Positive linear maps and spreads of matrices, Amer. Math. Monthly 121 (2014), 619-624.

7. E. Jiang and X. Zhan, Lower bounds for the spread of a Hermitian matrix, Linear Algebra Appl. 256 (1997), 153-163.

8. C. R. Johnson, R. Kumar and H. Wolkowicz, Lower bounds for the spread of a matrix, Linear Algebra Appl. 29 (1985), 161-173. 
9. L.V. Kantorovich, Functional analysis and applied mathematics, Translated by C. D. Benster. NBS Rep. 1509. U. S. Department of Commerce, National Bureau of Standards, Los Angeles, Calif., (1952).

10. M.A. Krasnoselskii and S.G. Krein, An iteration process with minimal residuals [in Russian], Mat. Sbornik N.S. 31 (1952), 315-334.

11. J.K. Merikoski and R. Kumar, Characterizations and lower bounds for the spread of a normal matrix, Linear Algebra Appl. 364 (2003), 13-31.

12. L. Mirsky, The spread of a matrix, Mathematika 3 (1956), 127-130.

13. L. Mirsky, Inequalities for normal and Hermitian matrices, Duke Math. J. 24 (1957), 591598.

14. M.S. Moslehian, Recent developments of the operator Kantorovich inequality, Expo. Math. 30 (2012), no. 4, 376-388.

15. T. Popoviciu, Sur les équations algébriques ayant toutes leurs racines réelles, Mathematica 9 (1935), 129-145.

16. R. Sharma, M. Gupta and G. Kapoor, Some better bounds on the variance with applications, J. Math. Inequal. 4 (2010), 355-363.

17. R. Sharma and R. Kumar, Remark on upper bounds for the spread of a matrix, Linear Algebra Appl. 438 (2013), 4359-4362.

18. H. Wolkowicz and G.P.H. Styan, Bounds for eigenvalues using traces, Linear Algebra Appl. 29 (1980), 471-506.

1 Department of Mathematics, Himachal Pradesh University, Shimla, India.

E-mail address: rajesh.sharma.hpn@nic.in; rajesh_hpu_math@yahoo.co.in

E-mail address: ravithakur345@yahoo.com

2 School of Basic Science, Bahra University, Wakanaghat, Solan, H.P., India. E-mail address: shalini.garga@yahoo.co.in 